WORKSHOP ON EDUCATIONAL INNOVATION

IN ARCHITECTURE JIDA'21

JORNADES SOBRE INNOVACIÓ

DOCENT EN ARQUITECTURA JIDA'21

ESCUELA TÉCNICA SUPERIOR DE ARQUITECTURA DE VALLADOLID 11 Y 12 DE NOVIEMBRE DE 2021 
Organiza e impulsa GILDA (Grupo para la Innovación y Logística Docente en la Arquitectura), en el marco del proyecto RIMA (Investigación e Innovación en Metodologías de Aprendizaje), de la Universitat Politècnica de Catalunya - BarcelonaTech (UPC) y el Institut de Ciències de l'Educació (ICE). http://revistes.upc.edu/ojs/index.php/JIDA

\section{Editores}

Daniel García-Escudero, Berta Bardí i Milà

\section{Revisión de textos}

Alba Arboix, Jordi Franquesa, Joan Moreno

\section{Edita}

Iniciativa Digital Politècnica Oficina de Publicacions Acadèmiques Digitals de la UPC

ISBN 978-84-9880-969-5 (IDP-UPC)

elSSN 2462-571X

(c) de los textos y las imágenes: los autores

(C) de la presente edición: Iniciativa Digital Politècnica Oficina de Publicacions

Acadèmiques Digitals de la UPC

Esta obra está sujeta a una licencia Creative Commons:

Reconocimiento - No comercial - SinObraDerivada (cc-by-nc-nd):

http://creativecommons.org/licences/by-nc-nd/3.0/es

https://creativecommons.org/licenses/by-nc-nd/4.0/

Cualquier parte de esta obra se puede reproducir sin autorización

pero con el reconocimiento y atribución de los autores.

No se puede hacer uso comercial de la obra y no se puede alterar, transformar o hacer obras derivadas. 


\section{JIDA}

\section{Comité Organizador JIDA'21}

\section{Dirección y edición}

\section{Berta Bardí i Milà (UPC)}

Dra. Arquitecta, Departamento de Proyectos Arquitectónicos, ETSAB-UPC

Daniel García-Escudero (UPC)

Dr. Arquitecto, Departamento de Proyectos Arquitectónicos, ETSAB-UPC

\section{Organización}

\section{Nieves Fernández Villalobos (UVA)}

Dra. Arquitecta, Teoría de la Arquitectura y Proyectos Arquitectónicos, ETSAVA

Jordi Franquesa (UPC)

Dr. Arquitecto, Departamento de Urbanismo y Ordenación del Territorio, ETSAB-UPC Joan Moreno Sanz (UPC)

Dr. Arquitecto, Departamento de Urbanismo y Ordenación del Territorio, ETSAB-UPC, ETSAV-UPC

\section{Gemma Ramón-Cueto (UVA)}

Dra. Arquitecta, Construcciones Arquitectónicas, Ingeniería del Terreno y Mecánica de los Medios continuos y Teoría de Estructuras, Secretaria Académica ETSAVA

Jorge Ramos Jular (UVA)

Dr. Arquitecto, Teoría de la Arquitectura y Proyectos Arquitectónicos, ETSAVA

Judit Taberna (UPC)

Arquitecta, Departamento de Representación Arquitectónica, ETSAB-UPC

\section{Coordinación}

\section{Alba Arboix}

Dra. Arquitecta, Teoría e Historia de la Arquitectura y Técnicas de la Comunicación, ETSAB-UPC

\section{Comunicación}

Eduard Llorens i Pomés

ETSAB-UPC 


\section{JIDA}

\section{Comité Científico JIDA'21}

Luisa Alarcón González

Dra. Arquitecta, Proyectos Arquitectónicos, ETSA-US

Eusebio Alonso García

Dr. Arquitecto, Teoría de la Arquitectura y Proyectos Arquitectónicos, ETSAVA-UVA

Darío Álvarez Álvarez

Dr. Arquitecto, Teoría de la Arquitectura y Proyectos Arquitectónicos, ETSAVA-UVA

Antonio Álvaro Tordesillas

Dr. Arquitecto, Urbanismo y Representación de la Arquitectura, ETSAVA-UVA

Atxu Amann Alcocer

Dra. Arquitecta, Ideación Gráfica Arquitectónica, ETSAM-UPM

Javier Arias Madero

Dr. Arquitecto, Construcciones Arquitectónicas, ETSAVA-UVA

Irma Arribas Pérez

Dra. Arquitecta, Diseño, Instituto Europeo de Diseño, IED Barcelona

Raimundo Bambó

Dr. Arquitecto, Urbanismo y ordenación del territorio, EINA-UNIZAR

Iñaki Bergera

Dr. Arquitecto, Proyectos Arquitectónicos, EINA-UNIZAR

Jaume Blancafort

Dr. Arquitecto, Arquitectura y Tecnología de la Edificación, ETSAE-UPCT

Enrique Manuel Blanco Lorenzo

Dr. Arquitecto, Proyectos Arquitectónicos, Urbanismo y Composición, ETSAC-UdC

Raúl Castellanos Gómez

Dr. Arquitecto, Proyectos Arquitectónicos, ETSA-UPV

Nuria Castilla Cabanes

Dra. Arquitecta, Construcciones arquitectónicas, ETSA-UPV

David Caralt

Arquitecto, Universidad San Sebastián, Sede Concepción, Chile

Rodrigo Carbajal Ballell

Dr. Arquitecto, Proyectos Arquitectónicos, ETSA-US

Eva Crespo

Dra. Arquitecta, Tecnología de la Arquitectura, ETSAB-UPC

Silvia Colmenares

Dra. Arquitecta, Proyectos Arquitectónicos, ETSAM-UPM

Còssima Cornadó Bardón

Dra. Arquitecta, Tecnología de la Arquitectura, ETSAB-UPC

Eduardo Delgado Orusco

Dr. Arquitecto, Proyectos Arquitectónicos, EINA-UNIZAR

Carmen Díez Medina

Dra. Arquitecta, Composición, EINA-UNIZAR

Sagrario Fernández Raga

Dra. Arquitecta, Teoría de la Arquitectura y Proyectos Arquitectónicos, ETSAVA-UVA

Arturo Frediani Sarfati

Dr. Arquitecto, Proyectos, Urbanismo y Dibujo, EAR-URV 


\section{JIDA}

Jessica Fuentealba Quilodrán

Dra. Arquitecta, Departamento Diseño y Teoría de la Arquitectura, Universidad del Bio-Bío, Concepción, Chile

Noelia Galván Desvaux

Dra. Arquitecta, Urbanismo y Representación de la Arquitectura, ETSAVA-UVA

María Jesús García Granja

Arquitecta, Departamento de Arte y Arquitectura, eAM'-UMA

Pedro García Martínez

Dr. Arquitecto, Arquitectura y Tecnología de la Edificación, ETSAE-UPCT

Mariona Genís Vinyals

Dra. Arquitecta, BAU Centre Universitari de Disseny, UVic-UCC

Eva Gil Lopesino

Arquitecta, Proyectos Arquitectónicos, ETSAM-UPM

María González

Arquitecta, Proyectos Arquitectónicos, ETSA-US

Arianna Guardiola Víllora

Dra. Arquitecta, Mecánica de los Medios Continuos y Teoría de Estructuras, ETSA-UPV

David Hernández Falagán

Dr. Arquitecto, Teoría e historia de la arquitectura y técnicas de comunicación, ETSAB-UPC

José $M^{a}$ Jové Sandoval

Dr. Arquitecto, Teoría de la Arquitectura y Proyectos Arquitectónicos, ETSAVA-UVA

Íñigo Lizundia Uranga

Dr. Arquitecto, Construcciones Arquitectónicas, ETSA EHU-UPV

Carlos Labarta

Dr. Arquitecto, Proyectos Arquitectónicos, EINA-UNIZAR

Emma López Bahut

Dra. Arquitecta, Proyectos, Urbanismo y Composición, ETSAC-UdC

Juanjo López de la Cruz

Arquitecto, Proyectos Arquitectónicos, ETSA-US

Alfredo Llorente Álvarez

Dr. Arquitecto, Construcciones Arquitectónicas, Ingeniería del Terreno y Mecánicas de los Medios

Continuos y Teoría de Estructuras, ETSAVA-UVA

Magda Mària Serrano

Dra. Arquitecta, Proyectos Arquitectónicos, ETSAV-UPC

Cristina Marieta Gorriti

Dra. Arquitecta, Ingeniería Química y del Medio Ambiente, EIG UPV-EHU

Zaida Muxí Martínez

Dra. Arquitecta, Urbanismo y ordenación del territorio, ETSAB-UPC

David Navarro Moreno

Dr. Ingeniero de Edificación, Arquitectura y Tecnología de la Edificación, ETSAE-UPCT

Amadeo Ramos Carranza

Dr. Arquitecto, Proyectos Arquitectónicos, ETSA-US

Patricia Reus

Dra. Arquitecta, Arquitectura y Tecnología de la Edificación, ETSAE-UPCT

Silvana Rodrigues de Oliveira

Dra. Arquitecta, Proyectos Arquitectónicos, ETSA-US 
Carlos Rodríguez Fernández

Dr. Arquitecto, Teoría de la Arquitectura y Proyectos Arquitectónicos, ETSAVA-UV

Jaume Roset Calzada

Dr. Físico, Física Aplicada, ETSAB-UPC

Borja Ruiz-Apilánez Corrochano

Dr. Arquitecto, UyOT, Ingeniería Civil y de la Edificación, EAT-UCLM

Patricia Sabín Díaz

Dra. Arquitecta, Proyectos Arquitectónicos, Urbanismo y Composición, ETSAC-UdC

Mara Sánchez Llorens

Dra. Arquitecta, Ideación Gráfica Arquitectónica, ETSAM-UPM

Luis Santos y Ganges

Dr. Urbanista, Urbanismo y Representación de la Arquitectura, ETSAVA-UVA

Carla Sentieri Omarrementeria

Dra. Arquitecta, Proyectos Arquitectónicos, ETSA-UPV

Marta Serra Permanyer

Dra. Arquitecta, Teoría e Historia de la Arquitectura y Técnicas de la Comunicación, ETSAV-UPC

Sergio Vega Sánchez

Dr. Arquitecto, Construcción y Tecnologías Arquitectónicas, ETSAM-UPM

José Vela Castillo

Dr. Arquitecto, Culture and Theory in Architecture and Idea and Form, IE School of Architecture and

Design, IE University, Segovia

Ferran Ventura Blanch

Dr. Arquitecto, Proyectos Arquitectónicos, eAM'-UMA

Isabel Zaragoza de Pedro

Dra. Arquitecta, Representación Arquitectónica, ETSAB-UPC 


\section{ÍNDICE}

1. Hábitat, paisaje e infraestructura en el entorno de la presa de El Grado (Huesca) Habitat, landscape and infrastructure in the surroundings of El Grado dam (Huesca). Estepa Rubio, Antonio; Elía García, Santiago.

2. Aprendiendo a dibujar confinados: un método, dos entornos. Learning to draw in confinement: one method, two environments. Salgado de la Rosa, María Asunción; Raposo Grau, Javier Fco, Butragueño Díaz-Guerra, Belén.

3. Aprendizaje basado en proyecto en la arquitectura a través de herramientas online. Project-based learning in architecture through online tools. Oregi, Xabat; Rodriguez, Iñigo; Martín-Garín, Alexander.

4. Técnicas de animación para la comprensión y narración de procesos de montaje constructivos. Animation techniques for understanding and storytelling of construction assembly processes. Maciá-Torregrosa, María Eugenia.

5. Desarrollo del Programa de Aprendizaje y Servicio en diversas asignaturas del grado de arquitectura. Development of the Learning and Service Program in various subjects of the degree of architecture. Coll-Pla, Sergio; Costa-Jover, Agustí.

6. Integración de estándares sostenibles en proyectos arquitectónicos. Integration of sustainable standards in architectural projects. Oregi, Xabat.

7. La Olla Común: una etnografía arquitectónica. The Common Pot: an architectural ethnography. Abásolo-Llaría, José.

8. Taller vertical, diseño de hábitat resiliente indígena: experiencia docente conectada. Vertical workshop, indigenous resilient habitat design: connected teaching experience. Lobato-Valdespino, Juan Carlos; Flores-Romero, Jorge Humberto.

9. Lecciones espaciales de las instalaciones artísticas. Learning from the space in art installations. Zaparaín-Hernández, Fernando; Blanco-Martín, Javier.

10. Alternativas para enseñar arquitectura: del proyecto introspectivo al campo expandido. Alternatives for Teaching Architecture: From the Introspective Project to the Expanded Field. Juarranz Serrano, Angela; Rivera Linares, Javier.

11. Una Herramienta de apoyo a la Docencia de las Matemáticas en los Estudios de Arquitectura. A Tool to support the Teaching of Mathematics for the Degree in Architectura. Reyes-Iglesias, María Encarnación.

12. Luvina, Juan Rulfo: materia de proyecto. Luvina, Juan Rulfo: matter of project. Muñoz-Rodríguez, Rubén; Pastorelli-Paredes, Giuliano. 
13. No se trata de ver videos: métodos de aprendizaje de la geometría descriptiva. It's not about watching videos: descriptive geometry learning methods. Álvarez Atarés, Fco. Javier.

14. Integration of Art-Based Research in Design Curricula. Integración de investigación basada en el arte en programas de diseño. Paez, Roger; Valtchanova, Manuela.

15. ¿Autómatas o autónomas? Juegos emocionales para el empoderamiento alineado y no alienado. Automata or autonomous? Emotional games for aligned and non-alienated empowerment. Ruiz Plaza, Angela.

16. Otras agendas para el estudiante. Another student agendas. Minguito-García, Ana Patricia.

17. Los Archivos de Arquitectura: una herramienta para la docencia con perspectiva de género. The Archives of Architecture: a tool for teaching with a gender perspective. Ocerin-Ibáñez, Olatz; Rodríguez-Oyarbide, Itziar.

18. Habitar 3.0: una estrategia para (re)pensar la arquitectura. Inhabiting 3.0: a strategy to (re)think architecture. González-Ortiz, Juan Carlos.

19. Actividades de aprendizaje para sesiones prácticas sobre la construcción en arquitectura. Learning activities for practical sessions about construction in architecture. Pons-Valladares, Oriol.

20. Getaria 2020: inspirar, pintar, iluminar. Getaria 2020: inspire, paint, enlight. Mujika-Urteaga, Marte; Casado-Rezola, Amaia; Izkeaga-Zinkunegi, Jose Ramon.

21. Aprendiendo a vivir con los otros a través del diseño: otras conversaciones y metodologías. Learning to live with others through design: other conversations and methodologies. Barrientos-Díaz, Macarena; Nieto-Fernández, Enrique.

22. Geogebra para la enseñanza de la Geometría Descriptiva: aplicación para la docencia online. Geogebra for the teaching of Descriptive Geometry: application for online education. Quintilla Castán, Marta; Fernández-Morales, Angélica.

23. La crítica bypass: un taller experimental virtual. The bypass critic: a virtual experimental workshop. Barros-Di Giammarino, Fabián.

24. Urbanismo táctico como herramienta docente para transitar hacia una ciudad cuidadora. Tactical urbanism as a teaching tool for moving towards a caring city. Telleria-Andueza, Koldo; Otamendi-Irizar, Irati.

25. Proyectos orales. Oral projects. Cantero-Vinuesa, Antonio.

26. Intercambios docentes online: una experiencia transdisciplinar sobre creación espacial. Online teaching exchanges: a transdisciplinary experience on spatial creation. Llamazares Blanco, Pablo. 
27. Nuevos retos docentes en geometría a través de la cestería. New teaching challenges in geometry through basketry. Casado-Rezola, Amaia; SanchezParandiet, Antonio; Leon-Cascante, Iñigo.

28. Mecanismos de evaluación a distancia para asignaturas gráficas en Arquitectura. Remote evaluation mechanisms for graphic subjects in architecture. Mestre-Martí, María; Muñoz-Mora, Maria José; Jiménez-Vicario, Pedro M.

29. El proceso didáctico en arquitectura es un problema perverso: la respuesta, un algoritmo. The architectural teaching process is a wicked problema: the answer, an algorithm. Santalla-Blanco, Luis Manuel.

30. La experiencia de habitar de los estudiantes de nuevo ingreso: un recurso docente. The experience of inhabiting in new students: a teaching resource. Vicente-Gilabert, Cristina; López Sánchez, Marina.

31. Habitar la Post·Pandemia: una experiencia docente. Inhabiting the Post-Pandemic: a teaching experience. Rivera-Linares, Javier; Ábalos-Ramos, Ana; Domingo-Calabuig, Débora; Lizondo-Sevilla, Laura.

32. El arquitecto ciego: método Daumal para estudiar el paisaje sonoro en la arquitectura. The blind architect: Daumal method to study the soundscape in architecture. Daumal-Domènech, Francesc.

33. Reflexión guiada como preparación previa a la docencia de instalaciones en Arquitectura. Guided reflection in preparation for the teaching of facilities in Architecture. Aguilar-Carrasco, María Teresa; López-Lovillo, Remedios María.

34. PhD: Grasping Knowledge Through Design Speculation. PhD: acceder al conocimiento a través de la especulación proyectual. Bajet, Pau.

35. andamiARTE: la Arquitectura Efímera como herramienta pedagógica. ScaffoldART: ephemeral Architecture as a pedagogical tool. Martínez-Domingo, Yolanda; Blanco-Martín, Javier.

36. Como integrar la creación de una biblioteca de materiales en la docencia. How to integrate the creation of a materials library into teaching. Azcona-Uribe, Leire.

37. Acciones. Actions. Gamarra-Sampén, Agustin; Perleche-Amaya, José Luis.

38. Implementación de la Metodología BIM en el Grado en Fundamentos de Arquitectura. Implementation of BIM Methodology in Bachelor's Degree in Architecture. Leon-Cascante, Iñigo; Uranga-Santamaria, Eneko Jokin; RodriguezOyarbide, Itziar; Alberdi-Sarraoa, Aniceto.

39. Cartografía de Controversias como recurso para analizar el espacio habitado. Mapping Controversies as a resourse for analysing the inhabited space. EspañaNaveira, Paloma; Morales-Soler, Eva; Blanco-López, Ángel. 
40. Percepciones sobre la creatividad en el Grado de Arquitectura. Perceptions on creativity at the Architecture Degree. Bertol-Gros, Ana; López, David.

41. El paisajismo en la redefinición del espacio público en el barrio de San Blas, Madrid. The landscape architecture in the redefinition of public space in the neighbourhood of San Blas, Madrid. Del Pozo, Cristina; Jeschke, Anna Laura.

42. De las formas a los flujos: aproximación a un proyecto urbano [eco]sistémico. Drawing throught a screen: teaching architecture in a digital world. CrosasArmengol, Carles; Perea-Solano, Jorge; Martí-Elias, Joan.

43. Dibujar a través de una pantalla: la enseñanza de la arquitectura en un mundo digital. Drawing throught a screen: teaching architecture in a digital world. Alonso-Rodríguez, Marta; Álvarez-Arce, Raquel.

44. Land Arch: el arte de la tierra como Arquitectura, la Arquitectura como arte de la tierra. Land Arch: Land Art as Architecture, Architecture as Land Art. ÁlvarezAgea, Alberto; Pérez-de la Cruz, Elisa.

45. Hyper-connected hybrid educational models for distributed learning through prototyping. Modelo educacional híbrido hiperconectado para el aprendizaje mediante creación de prototipos. Chamorro, Eduardo; Chadha, Kunaljit.

46. Ideograma. Ideogram. Rodríguez-Andrés, Jairo; de los Ojos-Moral, Jesús; FernándezCatalina, Manuel.

47. Taller de las Ideas. Ideas Workshop. De los Ojos-Moral, Jesús; Rodríguez-Andrés, Jairo; Fernández-Catalina, Manuel.

48. Los proyectos colaborativos como estrategia docente. Collaborative projects as a teaching strategy. Vodanovic-Undurraga, Drago; Fonseca-Alvarado, MaritzaCarolina; Noguera- Errazuriz, Cristóbal; Bustamante-Bustamante, Teresita-Paz.

49. Paisajes Encontrados: docencia remota y pedagogías experimentales confinadas. Found Landscapes: remote teaching and experimental confined pedagogies. Prado Díaz, Alberto.

50. Urbanismo participativo: una herramienta docente para tiempos de incertidumbre. Participatory urban planning: a teaching tool for uncertain times. Carrasco i Bonet, Marta; Fava, Nadia.

51. El portafolio como estrategia para facilitar el aprendizaje significativo en Urbanismo. Portfolio as a strategy for promoting meaningful learning in Urbanism. Márquez-Ballesteros, María José; Nebot-Gómez de Salazar, Nuria; Chamizo-Nieto, Francisco José.

52. Participación activa del estudiante: gamificación y creatividad como estrategias docentes. Active student participation: gamification and creativity as teaching strategies. Loren-Méndez, Mar; Pinzón-Ayala, Daniel; Alonso-Jiménez, Roberto F. 


\section{JIDA}

53. Cuaderno de empatía: una buena práctica para conocer al usuario desde el inicio del proyecto. Empathy workbook - a practice to better understand the user from the beginning of the project. Cabrero-Olmos, Raquel.

54. Craft-based methods for robotic fabrication: a shift in Architectural Education. Métodos artesanales en la fabricación robótica: una evolución en la experiencia docente. Mayor-Luque, Ricardo; Dubor, Alexandre; Marengo, Mathilde.

55. Punto de encuentro interdisciplinar: el Museo Universitario de la Universidad de Navarra. Interdisciplinary meeting point. The University Museum of the University of Navarra. Tabera Roldán, Andrés; Velasco Pérez, Álvaro; Alonso Pedrero, Fernando.

56. Arquitectura e ingeniería: una visión paralela de la obra arquitectónica. Architecture and engineering: a parallel vision of architectural work. GarcíaAsenjo Llana, David.

57. Imaginarios Estudiantiles de Barrio Universitario. Student's University Neighborhood Imaginaries. Araneda-Gutiérrez, Claudio; Burdiles-Allende, Roberto; Morales-Rebolledo Dehany.

58. El aprendizaje del hábitat colectivo a través del seguimiento del camino del refugiado. Learning the collective habitat following the refugee path. CastellanoPulido, F. Javier.

59. El laboratorio de investigación como forma de enseñanza: un caso de aprendizaje recíproco. The research lab as a form of teaching: a case of reciprocal learning. Fracalossi, Igor. 


\title{
Percepciones sobre la creatividad en el Grado de Arquitectura
}

\section{Perceptions on creativity at the Architecture Degree}

\author{
Bertol-Gros, Ana ${ }^{a}$; López, David ${ }^{b}$ \\ a Escuela de Arquitectura, Universidad San Jorge, Zaragoza y doctoranda Universitat Politècnica de \\ Catalunya, Barcelona. abertol@usj.es; b Departamento de Arquitectura de Computadores. Universitat \\ Politècnica de Catalunya, Barcelona. david@ac.upc.edu
}

\begin{abstract}
In a world dominated by standardization, the future of humanity depends on educating creative people. An original, useful and valuable idea cannot be obtained through standardized processes or an internet search. This communication collects the results of an investigation carried out with focus groups, consisting of two groups of students (Junior and Senior) and a group of teachers, from the Architecture Degree at San Jorge University. The research aim was to learn about the participant's percetions about creativity and discover what attitudes and methods encourage or impede its development. Open questions about the topic were posed to rise discussions among participants. Afterwards, the dialogues were analyzed according to the three keys to developing creativity in the classroom proposed by Robert Sternberg: generating opportunities, fostering creativity and rewarding the creative effort.
\end{abstract}

Keywords: creativity in architecture studies, perception of creativity, educational methodologies.

Thematic areas: architecture degree, self-regulated learning, research on education.

\begin{abstract}
Resumen
En un mundo dominado por la estandarización, el futuro de la humanidad depende de educar personas creativas. Una idea original, útil y con valor, no se puede obtener mediante procesos estandarizados ni una búsqueda en internet. Esta comunicación recoge los resultados de una investigación mediante grupos focales, realizada con dos grupos de estudiantes (Junior y Senior) y un grupo de docentes, del Grado de Arquitectura de la Universidad San Jorge. El objetivo de la investigación era conocer las percepciones que los participantes tienen sobre la creatividad y descubrir qué actitudes y métodos fomentan o impiden el desarrollo de ésta. Se plantearon preguntas abiertas sobre el tema para generar diálogo entre los participantes. Posteriormente se analizaron las respuestas según las tres claves para desarrollar la creatividad en el aula propuestas por Robert Sternberg: generar oportunidades, fomentar la creatividad y recompensar el esfuerzo creativo.
\end{abstract}

Palabras clave: creatividad en los estudios de arquitectura, percepción de la creatividad, metodologías educativas.

Bloques temáticos: grado de arquitectura, metodología de autoregulación del aprendizaje, investigación educativa. 


\section{Introducción}

La creatividad ha sido definida como una competencia esencial del siglo XXI.

En todo el mundo, los responsables políticos y líderes empresariales han reconocido que se debe promover una nueva forma de pensar a través de la educación para desarrollar soluciones a problemas complejos: como el calentamiento global, migraciones, o futuras pandemias (IBM, 2010; OECD, 2019; United Nations, 2015; World Economic Forum, 2020). La creatividad brinda a los estudiantes la capacidad de ver las cosas desde nuevas perspectivas, generar ideas originales y útiles, plantear nuevas preguntas y encontrar soluciones a problemas complejos (Plucker et al., 2004; Sternberg y Lubart, 1991). Por eso, la creatividad se considera una competencia crucial en todos los niveles, desde la educación primaria hasta la terciaria (OECD, 2019).

La creatividad es un concepto complejo y multidimensional que puede manifestarse de diferentes formas. Estudios bibliográficos (Mejia et al., 2021; Shubina y Kulakli, 2020) ponen de manifiesto que el campo de investigación está muy fragmentado $y$, a menudo, es inconsistente: la producción creativa se ha confundido a veces con la capacidad creativa y las habilidades de pensamiento divergente con el constructo general de la creatividad (Fusi et al., 2020). El concepto moderno de creatividad fue acuñado por Paul Guilford, en su discurso de la American Psychologist Asociation (Guilford, 1950), donde evidenció la falta de creatividad presente en el sistema educativo. Desde entonces se ha trabajado para encontrar una definición de un concepto difícil de acotar y entender: "La creatividad necesita de sí misma para explicarse" (Still y d'Inverno, 2016). Al no disponer de una definición consensuada universalmente, y por ello continuar siendo un concepto abstracto, su introducción en el sistema educativo sigue siendo difusa.

Hay varias formas de abordar el desarrollo de una definición de la creatividad. Es comúnmente aceptado considerar la creatividad desde cuatro puntos de vista diferentes: Persona, Proceso, Producto y Prensa (Torrance, 1993). Estas cuatro perspectivas fueron propuestas en el "modelo de las 4P de la creatividad" (Rhodes, 1961). Las 4P representan la naturaleza de las Personas creativas, su inteligencia y personalidad; los Procesos que utilizan, las metodologías que la implementan u obstaculizan; los Productos o el resultado final de una innovación, sea un objeto o idea; y la Prensa o el entorno que apoya u obstaculiza la creatividad, con conceptos como la motivación y el sentido de identidad del grupo.

¿Favorecen los estudios de grado de Arquitectura este concepto de "Prensa"? Está ampliamente demostrado que lo que determina un entono idóneo para potenciar la creatividad es la motivación intrínseca o extrínseca y el entorno social (Amabile, 2011). En esta investigación se estudian las percepciones sobre el entorno creativo del Grado de Arquitectura. Para ello se crearon unos grupos focales con alumnos y profesores para conocer sus percepciones sobre los factores que promueven u obstaculizan la creatividad en el aula.

\section{Objetivos del estudio}

La creatividad está definida, promovida (o bloqueada) y motivada por el entorno donde se produce. Algunos entornos sociales promueven más los cambios que otros, o están más dispuestos a aceptarlos. La mentalidad abierta del entorno también depende de la persona que genera la novedad, los efectos que pueda provocar el cambio y el campo en el que queramos introducirlo. Además, el entorno no es simplemente pasivo, aceptando o no el cambio, sino que 
influye en la cantidad y el tipo de novedad que se genera en primer lugar (A. Cropley y Cropley, 2009).

Numerosas investigaciones han abordado el estudio de las condiciones y actitudes del entorno para fomentar el desarrollo de la creatividad (Amabile, 2011; Baer y Kaufman, 2004; A. Cropley y Cropley, 2009; Hennessey, 2019; Kazerounian y Foley, 2007; Sternberg, 2007). Esta investigación se ha centrado en las tres bases que propone Robert Sternberg, para desarrollar la competencia creativa en el entorno educativo: 1) hay que ofrecer actividades donde se pueda desarrollar la creatividad; 2) hay que animar al estudiantado a participar en dichas tareas; y finalmente, 3) hay que recompensar al estudiantado cuando responde al estímulo y se comporta de manera creativa. "Se necesitan las tres, quite las oportunidades, el ánimo, o las recompensas, y estará quitando la creatividad" (Sternberg, 2007).

Primero, los estudiantes deben tener la oportunidad de desarrollar la creatividad. Para generar esa oportunidad hay que hacer espacio en el aula, los docentes debemos fomentar la libertad de los estudiantes para proponer, crear y expresar. Los estudiantes necesitan participar en proyectos suficientemente abiertos y flexibles. Se les debe alentar a hacer preguntas, no dar por sentado, y cuestionarse los problemas tal como se les presenta (D. Cropley, 2015).

En segundo lugar, los estudiantes deben ser animados a participar en tareas creativas. Hay que permitir que los estudiantes vean que pueden ser creativos y eviten el pensamiento equivocado de: "no soy creativo" (Robinson, 2011). Exigir la creatividad como parte del trabajo permite a los estudiantes ver que ser creativo es algo positivo para su formación. Esto requiere que los profesores comprendan la creatividad, cómo se manifiesta en los productos de arquitectura, y alentar a los estudiantes a incorporarlo en su trabajo. Para promover la creatividad, tenemos que demostrar nuestra propia flexibilidad, apertura, tolerancia a la ambigüedad y resiliencia. Necesitamos demostrar que entendemos qué es la creatividad, por qué es valiosa y por qué debería estar presente en los planes de estudios (D. Cropley, 2015; Sternberg, 2007).

En tercer lugar, los estudiantes deben ser recompensados cuando responden al estímulo y se comportan de manera creativa. La retroalimentación y la motivación son un factor clave para promover el aprendizaje (Bueno, 2019; Hattie y Timperley, 2007; Martín, 2020). Para que la retroalimentación ${ }^{1}$ desarrolle su mayor potencial, es importante que el alumno no tenga miedo de recibirlo. Para conseguir que los alumnos hagan del feedback un aliado de su aprendizaje, resultará fundamental trabajar sus creencias y hablarles abiertamente sobre la naturaleza del error desde una perspectiva de mentalidad de crecimiento (Bueno, 2019; Dweck, 2017; Martín, 2020).

Para aprender sobre el error y el límite de los riesgos, los estudiantes deben recibir tareas desafiantes para desarrollar su resiliencia, se les debe dar la oportunidad de fallar y volver a intentarlo. Tienen que aprender qué riesgos son aceptables y cuáles no. También es necesario fomentar la sensación de que a veces es necesario trabajar un poco más para obtener la recompensa, hay que impulsar a los estudiantes al máximo de sus capacidades. Esto no significa aumentar el numero de actividades, sino que se profundice más tanto en competencias técnicas como profesionales (D. Cropley, 2015). Al fin y al cabo, para ser un arquitecto creativo, primero se debe ser técnicamente competente. La creatividad es diferente en cada dominio del conocimiento (Baer y Kaufman, 2004; Simonton, 2014), por eso para poder ser creativos se necesita una base teórica con la que poder operar.

\footnotetext{
${ }^{1}$ También usado su anglicismo, feedback
} 
Las emociones no son únicamente necesarias para el aprendizaje, sino también para el desarrollo de la creatividad. El proceso creativo requiere estar abierto al cambio, así como tolerar la ambigüedad y la complejidad. La tensión del proceso a menudo lleva a responder con frustración, desconfianza, impaciencia, ira o sarcasmo. Estas respuestas emocionales afectan la actitud de todos los involucrados y dificultan que el proceso creativo se desarrolle de manera efectiva (Murdock et al., 2007).

\section{Método}

Este trabajo se realizó por medio de grupos focales, una metodología de investigación cualitativa exploratoria. Método utilizado para descubrir los sentimientos, valores, actitudes, reacciones y experiencias de los participantes sobre un tema. Formados por grupos de entre 5 y 10 personas que comparten un conjunto particular de características o experiencias, éstas se reúnen bajo la guía de un moderador para discutir un tema de importancia para un estudio en particular (Gizir, 2007).

La lógica de la investigación cualitativa se basa en estrategias de muestreo "intencionales" diseñadas para mejorar la comprensión de las experiencias de los individuos seleccionados (Devers y Frankel, 2000). Primero se estudiaron las percepciones sobre la creatividad del estudiantado, para ello se decidió generar un marco de investigación dividiendo a los alumnos en dos grupos: los Senior, de últimos cursos del grado; y los Junior, de primeros cursos. Estos últimos llevan menos tiempo dentro del Grado de Arquitectura $^{2}$, por lo que sus percepciones se esperan lejos del bagaje arquitectónico. Las percepciones de los Senior se esperan más arraigadas en la cultura arquitectónica. Una vez hecha esta distinción se generaron dos grupos de estudiantes por medio de voluntariado. Estos grupos focales se realizaron, de forma presencial, a mediados del mes de junio del 2021, tras finalizar los exámenes en convocatoria ordinaria.

Tabla 1. Estudiantes

\begin{tabular}{ccc}
\hline Estudiante & Sexo & Curso \\
\hline 1: Senior & $\mathrm{F}$ & $3^{\circ}$ \\
2: Senior & $\mathrm{M}$ & $3^{\circ}$ \\
3: Senior & $\mathrm{F}$ & $4^{\circ}$ \\
4: Senior & $\mathrm{M}$ & $5^{\circ}$ \\
5: Senior & $\mathrm{M}$ & $5^{\circ}$ \\
6: Junior & $\mathrm{F}$ & $1^{\circ}$ \\
7: Junior & $\mathrm{M}$ & $1^{\circ}$ \\
8: Junior & $\mathrm{M}$ & $2^{\circ}$ \\
9: Junior & $\mathrm{M}$ & $2^{\circ}$ \\
10: Junior & $\mathrm{M}$ & $2^{\circ}$ \\
\hline
\end{tabular}

\footnotetext{
${ }^{2}$ Grado de la Universidad San Jorge (Zaragoza, España). Es un grado joven (creado en 2010) que se imparte en una universidad privada y cuenta con un reducido número de estudiantes, actualmente 40 alumnos repartidos en cinco cursos.
} 
Al concluir la investigación con los estudiantes, se vio preciso completar los resultados analizando la perspectiva del cuerpo docente. Por lo que se realizó otro grupo focal con éstos, que por motivos de organización se dividió en dos. El marco de investigación en este caso se diseñó emulando la composición del cuerpo docente del grado, así como las respuestas anteriormente recibidas por los alumnos. Estos grupos focales se realizaron, de forma mixta ${ }^{3}$, a principios del mes de julio del 2021, tras finalizar los exámenes en convocatoria extraordinaria.

Tabla 2. Docentes

\begin{tabular}{ccccc}
\hline Docente & Sexo & Experiencia & Bloque $^{4}$ & Dedicación \\
\hline 1 & M & 19 años & $\begin{array}{c}\text { Propedéutico }\left(1^{\circ}\right) \text {, Proyectual }\left(2^{\circ}\right) \text { y } \\
\text { Nuclear }\left(5^{\circ}\right)\end{array}$ & Completa \\
2 & M & 19 años & Técnico $\left(2^{\circ}, 3^{\circ}\right.$ y $\left.5^{\circ}\right)$ & Parcial \\
3 & $\mathrm{M}$ & 11 años & Proyectual $\left(2^{\circ}\right)$ y Nuclear $\left(4^{\circ}\right)$ & Completa \\
4 & $\mathrm{M}$ & 10 años & Técnico $\left(4^{\circ}\right)$ & Completa \\
5 & $\mathrm{~F}$ & 9 años & Proyectual $\left(2^{\circ}, 3^{\circ} \mathrm{y} 4^{\circ}\right)$ & Completa \\
6 & $\mathrm{~F}$ & 18 años & Proyectual $\left(2^{\circ}\right)$ & Completa \\
7 & $\mathrm{M}$ & 12 años & Nuclear $\left(2^{\circ}\right)$ y Proyectual $\left(3^{\circ}\right)$ & Parcial \\
8 & $\mathrm{M}$ & 9 años & Proyectual $\left(1^{\circ} \mathrm{y} 4^{\circ}\right)$ & Parcial \\
\hline
\end{tabular}

En los grupos focales, el moderador estructura unas preguntas abiertas para generar diálogo entre los entrevistados, produciendo así los datos cualitativos que brindan conocimientos sobre las actitudes, percepciones y opiniones de los participantes como un procedimiento de recolección de datos (Gizir, 2007).

Para entender la percepción de los estudiantes sobre la generación de oportunidades se plantearon preguntas como: ¿en qué materia sientes que te puedes expresar con más libertad? Y a los docentes: ¿cuál es tu actitud para generar libertad de expresión? Respecto al fomento de la creatividad se preguntó a los estudiantes: ¿creéis que la creatividad será importante en tu futuro laboral? Y para entender la perspectiva del profesorado: ¿creéis que es importante formar estudiantes creativos? Finalmente, sobre la recompensa de ser creativo, a los estudiantes se les plantearon preguntas como:¿alguna vez habéis podido aprender equivocándoos? o ¿en qué actividad habéis sentido más libertad para equivocaros? $\mathrm{Y}$ posteriormente, a los docentes: Cuando un estudiante se equivoca, ¿le explicáis el fallo?¿Cómo los motiváis a esforzarse y trabajar?

Tras realizar las entrevistas, donde las respuestas fueron grabadas y transcritas, se realizó un análisis sistemático siguiendo un proceso secuencial, objetivo y contrastado, buscando tendencias y patrones que reaparecieran dentro de uno o varios grupos focales.

\footnotetext{
${ }^{3}$ Dos docentes online y dos en presencial.

${ }^{4}$ Bloque Propedeútico: asignaturas de primer curso. Bloque Proyectual: asignaturas de proyectos, urbanismo y cultura arquitectónica. Bloque Nuclear: asignaturas de integración y optativas de quinto. Bloque Técnico: instalaciones, estructuras y construcción.
} 


\section{Resultados y discusión}

A continuación se comentan las respuestas más relevantes.

\subsection{Generar oportunidades}

En cuanto a las oportunidades para desarrollar la creatividad, los estudiantes responden que sienten libertad cuando los profesores aceptan sus propuestas y su forma de trabajar. Perciben la libertad para explorar cuanta más confianza muestra el docente en su trabajo; cuando se sienten cómodos e ilusionados por ir a clase; y cuando más miedo tienen al corregir, porque saben que se han arriesgado: "Cuando tenemos miedo es porque hemos hecho lo que a nosotros nos parece guay y tenemos miedo a que nos tumben algo que creemos que está bien, suele surgir por una concesión de libertad".

Los profesores, por el contrario, responden que promueven la libertad generando debates con los alumnos, dándoles la oportunidad de expresar sus opiniones y, así, su personalidad: "Converso con ellos sobre los temas que se están tratando, les pregunto qué opinan, les hago buscar noticias relacionadas con el tema en la prensa, sobre todo, que traigan casos que ellos conozcan, casos reales y que los compartan y lo expongan en clase". Se intuye que los profesores intentan generar libertad de expresión fomentando que los alumnos hablen y dialoguen, pero éstos lo que buscan es un sentimiento de confianza para poder explorar.

Tanto profesores como alumnos reflejan que en las asignaturas técnicas ${ }^{5}$ no sienten espacio para la libertad al tener una carga teórica fuerte. Los profesores achacan esta falta de libertad a que para explicar la teoría se necesita más tiempo, que es robado de la parte práctica, por lo que no tienen tiempo de experimentar, conversar con los alumnos y expresar con libertad: "En proyectos tenemos más tiempo y contamos muchas menos cosas. Al final en las asignaturas técnicas hay mucho menos tiempo y mucha más teoría. Entiendo que en una asignatura técnica es difícil que el alumno se exprese, porque el tiempo es un factor importante". Los alumnos, por su parte, achacan a las restricciones teóricas de las asignaturas técnicas la culpabilidad de no poder proponer con libertad, se sienten constreñidos por las leyes.

En cuanto a poder trabajar la ambigüedad y afrontar diferentes perspectivas, los Senior sí que perciben y practican la capacidad de proponer y afrontar problemas desde diferentes puntos de vista. Al contrario, los Junior sienten que, al no tener ni conocer todas las herramientas necesarias, no pueden proponer soluciones dispares por lo que habitúan a trabajar en su zona de confort, sobre todo en las asignaturas técnicas, porque tienen que seguir la teoría y eso no les da pie a experimentar: "En proyectos e integración ${ }^{6}$ sí que lo puedes afrontar de distintas maneras, pero al final en estructuras y construcción hay más una base teórica que es la que tienes que seguir, entonces ya no puedes tirar por otra perspectiva y te tienes que basar en lo que sabes y en las fórmulas". Una de las cosas que les paraliza a la hora de experimentar es que perciben incongruencias entre lo que les piden los profesores, lo que creen que deben hacer y lo que quieren proponer. Unos afirman: "Yo creo que tiran más hacia la técnica que hacia la creatividad" y otros les rebaten: "Los profesores deberían, en vez de tirar por lo poético, tirar más por lo lógico".

\footnotetext{
${ }^{5}$ Bloque Propedéutico 60 ECTS. Bloque Técnico 78 ECTS. Bloque Proyectual 114 ECTS. Bloque Nuclear 48 ECTS.

${ }^{6}$ Asignatura del bloque Nuclear que, como módulo vertebrador transversal, vertical y horizontalmente, busca explicar y orientar el contenido programático del resto de materias; $y$ también formará en el progreso madurativo del alumno como arquitecto en desarrollo desde su acceso a la Escuela. Este bloque representará la troncalidad formativa y permitirá anidar en ella cuantos contenidos y disciplinas externas al programa previo sea necesario incluir, facilitando la novedad y adecuación del programa entero a una realidad cambiante.
} 
Los docentes afirman que plantean diferentes perspectivas para trabajar y están abiertos a la ambigüedad, respuesta que coincide con la mayoría del alumnado. Profundizan en el tema comunicando una preocupación hacia el plan de estudios, sienten que es necesario que el grado cambie de perspectiva para poder dar paso a la experimentación y la creatividad. Afirman que hay que romper los límites de las asignaturas y enseñar arquitectura, en vez de construcción, estructuras o proyectos. Esta visión respalda la visión planteada por numerosos autores (Goldberg y Somerville, 2014; Jackson, 2014; López, 2021) sobre la necesidad de tener un plan de estudios holístico para fomentar, entre otras competencias, la creatividad.

La actitud actual frente a la creatividad queda reflejada en el comentario de este profesor: "A los alumnos no les puedes pedir que sean creativos en todas las asignaturas, porque son como diez trabajos y no tienen tiempo físico para hacerlos". Esto refleja una idea equivocada sobre la creatividad: ligar la capacidad creativa con la producción ${ }^{7}$. Centrarse demasiado en los detalles hace que pierdas la visión global del proyecto ${ }^{8}$, lo que impide el correcto funcionamiento de los detalles, por ser muchos; y del proyecto, por perder la perspectiva holística. Cargar a los estudiantes con numerosas entregas, pensando que así son creativos, solo está limitando su desarrollo. Visión compartida con otro docente: "Hay que hacerles entender que no son cosas estancas, conocimientos estancos y demás. Porque lo ven así, ellos salen por la puerta de clase de estructuras, entran a proyectos, les preguntas sobre la estructura y ¡no han pensado absolutamente nada!"

\subsection{Fomentar la creatividad}

En cuanto a la perspectiva de la creatividad, analizando las respuestas se observa una diferente perspectiva del concepto entre los dos grupos de estudiantes. Los Senior perciben la creatividad como esa picaresca o capacidad de ser resolutivo, como personas creativas nombran a compañeros suyos o a Gaudí, "por poner un ejemplo conocido". Mientras que los Junior transmiten una visión relacionada a conceptos visuales, estéticos o del genio creador. Nombran a Da Vinci, Beethoven o Miguel Ángel como ejemplos de personas creativas, o "Los Youtubers, tienen otra manera de creatividad, quizás más moderna que la pintura o la música". Esto refleja una falsa percepción sobre qué es la creatividad, por lo general asociada a grandes genios o "Big-C", cuando el sistema educativo debería desarrollar la creatividad del día a día: "Little-C y mini-C" (Kaufman y Beghetto, 2009).

La perspectiva del profesorado, aunque a priori dudan de tener estudiantes creativos, se asemeja a la de los Senior, con lo que podemos concluir que al final del grado los docentes consiguen transmitir su visión sobre la creatividad ligado a la técnica, teoría, resolución de problemas y el esfuerzo. La pregunta es, ¿les están transmitiendo la verdad sobre la creatividad o simplemente su perspectiva?

Preocupa que el profesorado refleje la visión de que la creatividad no se puede trabajar en asignaturas técnicas, con afirmaciones como "al final, es muy difícil en asignaturas técnicas ser creativo. Ellos no es que no quieran ser creativos. Quieren ser creativos donde creen que tienen que serlo y donde no, si les obligas a hacer un esfuerzo extra, se enfadan. Son creativos en proyectos e integración y a lo mejor un poco en urbanismo. No consideran que una asignatura

\footnotetext{
${ }^{7}$ Nombrado en la introducción como obstáculo para desarrollar la creatividad

${ }^{8}$ Micromanagement: es una forma negativa de gerenciar ejerciendo control excesivo sobre los detalles de un proyecto, perdiendo así la visión global y buen funcionamiento de éste.
} 
técnica pueda ser creativa". Las opiniones de los alumnos también reflejan esa visión: "Yo en clase de construcción o estructuras no tengo problema, porque dicen que está mal y lo asumes porque hay una base teórica". David Cropley (2015) desmiente esta falsa percepción y afirma que "el conocimiento técnico es la base de la creatividad en ingeniería", para ello explica las relaciones entre tipos de conocimiento, niveles de comprensión y formas de experiencia óptimas para desarrollar la creatividad.

La pregunta final de todos los grupos focales fue: ¿crees que la creatividad será importante en el futuro de la profesión de arquitectura? Los profesores rehuyeron dar una respuesta concisa, en cambio los alumnos sueñan con mundos digitales, construcciones espaciales, realidad virtual... ¿será verdad que las escuelas matan la creatividad? (Robinson, 2006).

\subsection{Recompensar el esfuerzo creativo}

En cuanto a la retroalimentación, los alumnos afirman que todo aprendizaje lo realizan a través de los errores, pero que echan en falta explicaciones, tacto y acompañamiento en el proceso de ese aprendizaje. Consideran que esta falta de inteligencia emocional por parte del cuerpo docente reduce mucho su nivel de motivación: "Igual que cuando dicen, no funciona y no dicen nada más. Falta mucha inteligencia emocional a nivel docente. Es que además me lo tiraban con tanta brusquedad que perdí la motivación para volver a pensar una cosa nueva". Es necesario que, primero docentes y luego estudiantes, sean formados sobre inteligencia emocional. La efectividad y calidad del proceso de enseñanza tienen relación con las competencias sociales y emocionales (Bueno, 2019; Pertegal-Felices et al., 2011).

Las respuestas de los docentes reflejan que, directa o indirectamente, relacionan la motivación con las calificaciones. Comparando las respuestas, los docentes intentan que sea el propio alumno quien se motive a través del dialogo, haciendoles ver cuáles son sus experiencias y objetivos y así sepan cómo esforzarse y trabajar: "Respecto a motivar a los estudiantes, es intentar que se miren al espejo. La motivación es buscar que ellos se motiven consigo mismos, que con sus propias experiencias y mochilas sean capaces de encontrar sus propios intereses". Es cierto que los estudiantes afirman que, en el taller vertical ${ }^{9}$, donde se les da la oportunidad de opinar, dialogar, y corregir entre compañeros y profesores, es donde más motivados se sienten y mejor aprenden de los errores. Pero echan en falta un mejor guiado en el resto de los formatos a la hora de aprender del error. Hay numerosas y similares opiniones por parte de los alumnos: "Respecto a lo de aprender de los errores es como que te suspenden, pero no te explican. 0 vale, si has aprobado con un 5 , se supone que me estás formando como arquitecto, voy a necesitar $X$ herramientas para mi carrera profesional, se supone que, si he sacado un 5 , el otro 5 no lo tengo". Está bien que el profesor consiga que el alumno tenga motivación intrínseca, pero la motivación extrínseca es igual de importante para fomentar la creatividad y el aprendizaje (Bueno, 2019; Martín, 2020).

Ante esta perspectiva, es interesante analizar las percepciones sobre la asignatura que mejor valoración recibe por parte de los alumnos "Hay como un estímulo constante, un feedback que te hace hacer las cosas y te emociona. El profesor también es brusco, pero no sé qué hace que yo me encontré motivado en casi todas las entregas, te valora mucho lo bueno y lo malo". Por su parte, el profesor comenta "Yo genero un clima de confianza, intento borrar un poco esa visión que ha habido siempre del profesor como un ente casi intocable, poniendo a todos al mismo

\footnotetext{
${ }^{9}$ Se refieren a la asignatura de Integración, explicada en la nota 4
} 
nivel, siempre manteniendo un poco los roles y el respeto. Intento romper barreras. Es un equilibrio un poco raro porque es mantener un poco el grado de profesor, el respeto y luego que ellos se sientan con capacidades de expresar libremente lo que sienten". Cuando la educación pone el foco en el estudiante, es cuando se produce un aprendizaje significativo (Bravo, 2020; Bueno, 2019).

\section{Limitaciones de la investigación}

Se deben tener presentes ciertas limitaciones del presente estudio. Primero, se ha trabajado sobre un entorno de una única universidad, por lo que su manera de trabajar puede sesgar los resultados; el estudio se debería ampliar en un futuro a otras universidades para poder generalizar las conclusiones. Habría que hacer un estudio en diferentes escuelas y grados, para definir qué es la creatividad en cada campo. Segundo, la selección de la muestra de los estudiantes por medio de una llamada voluntaria a la participación implica que los participantes tienen una personalidad dispuesta a la colaboración. Por último, la investigación y las entrevistas han sido realizadas por un miembro del cuerpo docente de la escuela, por lo que la relación con estudiantes y profesores puede haber influenciado los resultados obtenidos.

Pese a las limitaciones de esta investigación, los resultados siguen la línea de investigaciones similares con resultados muy interesantes (Hosseini, 2011; Rodgers y Jones, 2017; Taneri y Dogan, 2021). La investigación sobre creatividad no se está dando únicamente en diseño o arquitectura si no en otros campos como la medicina (Chan, 2013; Ku, 2015).

\section{Conclusión}

Se ha realizado una investigación mediante grupos focales para analizar las perspectivas de estudiantes y profesores de un mismo grado de arquitectura sobre la competencia creativa. Se han contrastado y analizado las respuestas ofrecidas por los entrevistados, buscando patrones, semejanzas y diferentes opiniones.

La dinámica general de las respuestas refleja la perspectiva apuntada por numerosos autores sobre que la sociedad tiene una idea equivocada acerca de la creatividad (Buhl, 1960; A. Cropley, 2016; D. Cropley, 2015; Kazerounian y Foley, 2007; Robinson, 2011; Walther y Radcliffe, 2007): Desde la percepción de que la creatividad es ser resolutivo, hasta la idea de que no puedes ser creativo en asignaturas técnicas. Al ser un concepto tan grande y abstracto, falta esfuerzo por entenderla. Como señalan estos autores, falta mucho trabajo de investigación sobre los límites de la creatividad y como implementarlos en curriculum universitario. No tiene que ser un trabajo individual sino guiado desde los planes de estudios (López, 2021).

El avance de este campo es imprescindible para la comunidad educativa, no solo en la formación de arquitectura si no en todos los campos STEAM. Esta investigación forma parte de una tesis doctoral que aspira a definir criterios de creatividad en asignaturas técnicas para poder introducirlo, de forma general y guiada, en los planes de estudios.

\section{Agradecimientos}

La presente investigación ha sido posible gracias a la participación y cooperación de alumnos y profesores del Grado de Arquitectura de la Universidad San Jorge. 


\section{Bibliografía}

AMABILE, T. (2011). Componential Theory of Creativity. In Encyclopedia of Management Theory. Harvard Business School. <https://doi.org/10.4135/9781452276090.n50>

BAER, J. y KAUFMAN, J.C. (2004). Bridging generality and specificity: The amusement park theoretical (apt) model of creativity. In J. C. Kaufman \& J. Baer (Eds.), Creativity across Domains: Faces of the Muse (1st ed., Issue 1, pp. 339-346). Taylor \& Francis Group. <https://doi.org/10.1080/02783190509554310>

BRAVO, J.A.F. (2020). Enseñar desde el cerebro del que aprende. Youtube. $<$ https://www.youtube.com/watch?v=mB7s7r7vafA>

BUENO, D. (2019). Neurociencia para educadores: Todo lo que los educadores siempre han querido saber sobre el cerebro de sus alumnos y nunca nadie se ha atrevido a explicárselo de manera comprensible y útil. Barcelona: Ediciones Octaedro.

BUHL, H.R. (1960). Creative engineering design. lowa State University Press.

CHAN, Z.C. (2013). Exploring creativity and critical thinking in traditional and innovative problem-based learning groups. Journal of Clinical Nursing, 22(15-16). <https://doi.org/10.1111/jocn.12186>

CROPLEY, A. (2016). The Myths of Heaven-Sent Creativity: Toward a Perhaps Less Democratic But More Down-to-Earth Understanding. Creativity Research Journal, 28(3). <https://doi.org/10.1080/10400419.2016.1195614>

CROPLEY, A. y CROPLEY, D. (2009). Fostering creativity: A diagnostic approach for higher education and organizations (Issue Hampton Huary). Hess. <http://www.emeraldinsight.com/10.1108/09544789410067943>

CROPLEY, D. (2015). Promoting creativity and innovation in engineering education. Psychology of Aesthetics, Creativity, and the Arts, 9(2), 161-171. <https://doi.org/10.1037/aca0000008>

DEVERS, K.J., y FRANKEL, R.M. (2000). Study design in Qualitative research 2: Sampling and data collection strategies. Education for Health, 13(2). <https://doi.org/10.1080/13576280050074543>

DWECK, C.S. (2017). Mindset: Changing the way you think to fulfil your potential. London: Hachette UK.

FUSI, G.; LAVOLPE, S.; CREPALDI, M.; y RUSCONI, M.L. (2020). The Controversial Effect of Age on Divergent Thinking Abilities: A Systematic Review. Journal of Creative Behavior, 55, 374-395. <https://doi.org/10.1002/jocb.461>

GIZIR, S. (2007). Focus groups in educational studies. Mersin Üniversitesi Eğitim Fakültesi Dergisi. $<$ https://doi.org/10.17860/efd.94890>

GOLDBERG, D.E. y SOMERVILLE, M. (2014). A whole new engineer. The coming revolution in Engineering Education. Douglas MI: Threejoy.

GUILFORD, J. P. (1950). Creativity. In American Psychologist (Vol. 5, Issue 9, pp. 444-454). American Psychological Association. <https://doi.org/10.1037/h0063487>

HATTIE, J. y TIMPERLEY, H. (2007). The power of feedback. In Review of Educational Research (Vol. 77, Issue 1). <https://doi.org/10.3102/003465430298487>

HENNESSEY, B.A. (2019). Motivation and creativity. In The Cambridge Handbook of Creativity: Second Edition. <https://doi.org/10.1017/cbo9780511807916.017>

HOSSEINI, A.S. (2011). University student's evaluation of creative education in universities and their impact on their learning. Procedia-Social and Behavioral Sciences, 15, 1806-1812. <https://doi.org/10.1016/j.sbspro.2011.04.007>

IBM. (2010). IBM 2010 Global CEO Study: creativity selected as most crucial factor for future success. <https://www.ibm.com/news/ca/en/2010/05/20/v384864m81427w34.html>

JACKSON, N. (2014). Developing students' creativity through a higher education. Proceedings from the International Symposium on "The Cultivation of Creativity in University Students," November 2013, 8-28.

KAUFMAN, J.C. y BEGHETTO, R.A. (2009). Beyond Big and Little: The Four C Model of Creativity. Review of General Psychology, 13(1), 1-12. <https://doi.org/10.1037/a0013688> 
KAZEROUNIAN, K. y FOLEY, S. (2007). Barriers to creativity in engineering education: A study of instructors and students perceptions. Journal of Mechanical Design, Transactions of the ASME, 129(7), 761-768. $<$ https://doi.org/10.1115/1.2739569>

KU, Y.L. (2015). Evaluating creative thinking of RN-BSN students in the course of clinical case study and practicum. Innovations in Education and Teaching International, 52(3). <https://doi.org/10.1080/14703297.2013.838144>

LÓPEZ, D. (2021). El futuro de los planes de estudios en Ingeniería Informática. Una visión (muy) personal. 6, 1-20. <https://upcommons.upc.edu/handle/2117/349447>

MARTíN, H.R. (2020). ¿Cómo aprendemos? Una aproximación científica al aprendizaje y la enseñanza (1ª). Editorial Graó.

MEJIA, C.; D'IPPOLITO, B.; y KAJIKAWA, Y. (2021). Major and recent trends in creativity research: An overview of the field with the aid of computational methods. Creativity and Innovation Management, 30(3), 475-497. <https://doi.org/10.1111/caim.12453>

MURDOCK, M.C.; CYNDI BURNETT; y MARIE MANCE. (2007). The Big Three-Understanding and Applying Basic Affective Skills that Underlie CPS: The Thinking Skills Model. 1-11.

OECD. (2019). OECD Future of Education and Skills 2030. OECD. <https://doi.org/10.1787/soc glance2019-en>

PERTEGAL-FELICES, M.L.; CASTEJÓN-COSTA, J.L.; y MARTíNEZ, M.Á. (2011). Competencias socioemocionales en el desarrollo profesional del maestro. Educacion XX1, 14(2). $<$ https://doi.org/10.5944/educxx1.14.2.253>

PLUCKER, J.A.; BEGHETTO, R.A.; y DOW, G.T. (2004). Why isn't creativity more important to educational psychologists? Potentials, pitfalls, and future directions in creativity research. Educational Psychologist, 39(2), 83-96. <https://doi.org/10.1207/s15326985ep3902 1>

RHODES, M. (1961). Analysis of Creativity. Can it be taught? Phi Delta Kappan, 42(7).

ROBINSON, K. (2011). Out of our minds: Learning to be creative. John Wiley \& Sons.

ROBINSON, K. (2006). Do schools kill creativity? TED. $<$ https://www.ted.com/talks/sir ken robinson do schools kill creativity/transcript>

RODGERS, P.A. y JONES, P. (2017). Comparing University Design Students' and Tutors' Perceptions of Creativity. Design Journal, 20(4). <https://doi.org/10.1080/14606925.2017.1323503>

SHUBINA, I. y KULAKLI, A. (2020). The Research Patterns of Creativity and Innovation: The Period of 20102019. International Journal of Emerging Technologies in Learning, 15(21), 89-102. <https://doi.org/10.3991/ijet.v15i21.16101>

SIMONTON, D.K. (2014). Hierarchies of Creative Domains. In The Philosophy of Creativity (pp. 247-262). Oxford University Press. <https://doi.org/10.1093/acprof:oso/9780199836963.003.0013>

STERNBERG, R.J. (2007). Creativity as a habit. Creativity: A Handbook for Teachers, c, 3-26. <https://doi.org/10.1142/9789812770868 0001>

STERNBERG, R.J. y LUBART, T.I. (1991). An Investment Theory of Creativity and Its Development. Human Development, 34(1), 1-31. <https://doi.org/10.1159/000277029>

STILL, A. y D'INVERNO, M. (2016). A history of creativity for future Al research. Proceedings of the 7th International Conference on Computational Creativity, ICCC 2016, 147-154.

TANERI, B. y DOGAN, F. (2021). How to learn to be creative in design: Architecture students' perceptions of design, design process, design learning, and their transformations throughout their education. Thinking Skills and Creativity, 39(January), 100781. <https://doi.org/10.1016/j.tsc.2020.100781>

TORRANCE, E.P. (1993). Understanding Creativity: Where to Start? Psychological Inquiry, 4(3), 232-234. <https://doi.org/10.1207/s15327965pli0403 17>

UNITED NATIONS. (2015). Transforming our world: The 2030 Agenda for Sustainable Development. <https://www.un.org/en/chronicle/article/goal-4-education-post-2015-sustainable-development-agenda>

WALTHER, J. y RADCLIFFE, D.F. (2007). The competence dilemma in engineering education: Moving beyond simple graduate attribute mapping. Australasian Journal of Engineering Education, 13(1), 41-51. <https://doi.org/10.1080/22054952.2007.11464000>

WORLD ECONOMIC FORUM. (2020). The future of Jobs report 2020. <http://www3.weforum.org/docs/WEF Future of Jobs 2020.pdf> 